\title{
Multivariate Chaotic Time Series Prediction Based on NARX Neural Networks
}

\author{
Yan Xiu* and Wei Zhang \\ School of science, Tianjin Chengjian University, Tianjin 300384, China \\ ${ }^{*}$ Corresponding author
}

\begin{abstract}
According to multivariate chaotic time series prediction problem, this paper establishes a multivariate chaotic time series forecasting model with nature structure import data based on NARX neural network. The simulation research of Lorenz chaotic time series proves that the forecasting precision of multivariate chaotic time series forecasting model with nature structure import data is much higher than using unvaried chaotic time series. And found the NARX neural network has strong nonlinear mapping ability than others. In comparison with other algorithms, the NARX modeling with nature structure import data method can make better predicting performance, thus it can be widely used in multivariate chaotic time series prediction.
\end{abstract}

Keywords-narx neural networks; chaotic time series; univariate; multivariate; prediction

\section{INTRODUCTION}

Chaotic time series forecasting methods and models is a hot research field in recent years, involves many subjects, is widely used in many fields. It has been widely applied in the wind speed forecasting, hydrological forecast, the movement accuracy of CNC machine tools forecast, wind power prediction and forecasting of financial [1-6]. So far, domestic and foreign scholars have done a lot of researches on chaotic prediction theory. They have obtained some valuable results by neural network, support vector machine prediction research, and the extreme learning machine model [7-11].

Among them, the neural network model has been widely used in the prediction of chaotic time series. Neural network is divided into static neural network and dynamic neural network. Static neural networks are commonly used in BP and RBF neural network, the network does not have the feedback and memory function, such that the network in the training or simulation that the current output is only related to the current input, has nothing to do with the past input and output. Due to the lack of feedback function and memory function, the generalization ability of the network is low, which is not suitable for chaotic time series prediction. Compared with static neural network, dynamic neural network is more suitable for chaotic systems. The characteristics of chaotic time series is the input and output of the past is closely associated with the current output, while the dynamic neural network with feedback and memory function, so the use of dynamic neural network prediction often can get more accurate results. NARX regression neural network is the most widely used dynamic nonlinear neural network. Its performance is generally better than the full regression neural network. Due to the inclusion of the NARX neural network multi step input and output delay, it can reflect the historical status of the information system. So the network has the memory function, and it has good nonlinear mapping ability, robustness and adaptability. It can better describe the characteristics of chaotic time series system.

At present, the prediction of chaotic time series is mainly based on single variable time series prediction and multivariate time series prediction. Most of that single variable chaotic time series for time series obtained in practice for finite length and noise, although the Taken 's embedding theory of phase space reconstruction of the original system got more information, but its prediction effect is not better than the effect of prediction of multivariate chaotic time series. This is due to the fact that the complex system is usually described by several variables, and more dynamic information can be obtained by using multivariate time series modeling. In many studies, the multivariate time series data through phase space reconstruction or other methods of input data for the conversion. But this paper thinks that the chaotic time series data's number is enough and no noise. By using its natural structure as the input of the model, the structure can better reflect the original chaotic system. So the paper directly uses the natural structure of the multivariate time series of chaotic system for modeling, and using the NARX neural network's powerful approximation fitting ability of multivariate chaotic time series prediction model of NARX neural network, in order to obtain better prediction effect.

\section{INTRODUCTION OF NARX NEURAL NETWORK}

NARX neural network is a nonlinear Auto-Regressive model with Exogenous Inputs [12]. A typical NARX regression neural network consists of input layer, hidden layer and output layer and the input and output delay, to determine the input and output delay order number, the number of hidden layer neurons prior before application. The schematic diagram of the network structure is shown in Figure I:

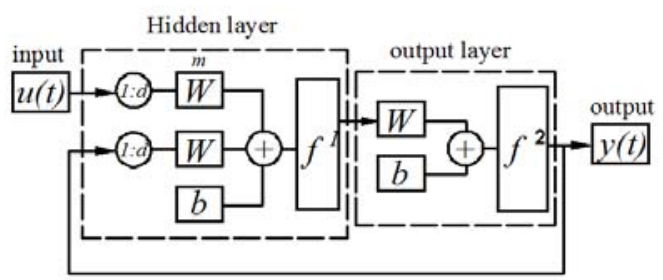

FIGURE I. NARX NEURAL NETWORK STRUCTURE 
In Figure I, the parameters of $d$ is order delay; the $\mathrm{m}$ is the number of neurons in the hidden layer; the $\mathrm{w}$ is the input vector of the network and the feedback vector weight matrix; the $b$ is bias; the $\mathrm{f}^{1}$ is neural network hidden layer activation function with the tansig function; the $\mathrm{f}^{2}$ is the neural network output layer activation function, using purelin transfer function.

The NARX neural network model is established by introducing the time delay module and the output feedback to establish the dynamic recurrent network. In the process of training, verification and testing, the input of the network model includes not only the original input data, but also the output data after training. Then the generalization ability of the network is improved.

The mathematical model of NARX neural network can be expressed as:

$$
y(t)=f\left[y(t-1), y(t-2), \ldots, y\left(t-n_{y}\right), u(t-1), u(t-2), \ldots, u\left(t-n_{u}\right)\right]
$$

The function $f(\cdot)$ represents the nonlinear function of neural network. It can be seen that the output data can be used as input in the feedback function and improve the accuracy of the neural network through open cycle training or closed loop training.

In order to achieve the desired results, it is necessary to select the parameters of the NARX neural network model. The function $f(\cdot)$ of the equations (1) can be trained and studied by the network, which depends on the number of neurons in the hidden layer. If the input layer data through multiple simple neurons of hidden layer, the output value is close to the given expected value, and the prediction error autocorrelation and input errors associated with the error map in a reasonable range by training NARX neural network, the applicability of NARX neural network model is better. If the error is large, you need to modify the number of neurons and order of delay in the hidden layer, until the output value and the expected value of the error is in the acceptable range. Therefore, the number of neurons and the number of delay orders need to be tested repeatedly to determine.

\section{MODEL EVALUATION CRITERIA}

In this paper, we use the root mean square error (RMSE) to measure the prediction error. The root mean square error is a method to describe the system error, which can be used to measure the deviation between the predicted data and the target data. Standard root mean square error (RMSE) is as follows:

$$
R M S E=\sqrt{\frac{\sum_{i=1}^{n}\left(y_{\text {true }, i}-y_{\text {predict }, i}\right)^{2}}{n}}
$$

In the formula, $y_{\text {true,i }}$ is the actual value, $y_{\text {predict,i }}$ is the predicted value, and $\mathrm{n}$ is the number of test samples.

\section{SIMULATION STUDY}

\section{A. Preparation of Chaotic Time Series Data}

In this paper, the classical chaotic time series Lorenz attractor is simulated to verify the validity of the proposed method. The Lorenz chaotic time series is defined as follows:

Lorenz attractor equation

$$
\begin{aligned}
& \dot{x}=\sigma(y-x), \\
& \dot{y}=r x-x z-y, \\
& \dot{z}=x y-b z
\end{aligned}
$$

Among them, the parameter values is set to $\sigma=16.0$, $\mathrm{r}=45.92, \mathrm{~b}=4.0$, and integral step $\mathrm{h}=0.01$. Using the four order Runge-Kutta method solves integral equations. Given the initial value $(-1,0,1)$ iteration produces a length of 32100 bit Lorenz chaotic system. In order to make the research of representative, we remove the time-series data in the initial position to the front part of the transient chaos, generating 2100 data points as the research sample, the first 2000 data as training set, 100 data as the test data set, as shown in Figure II.

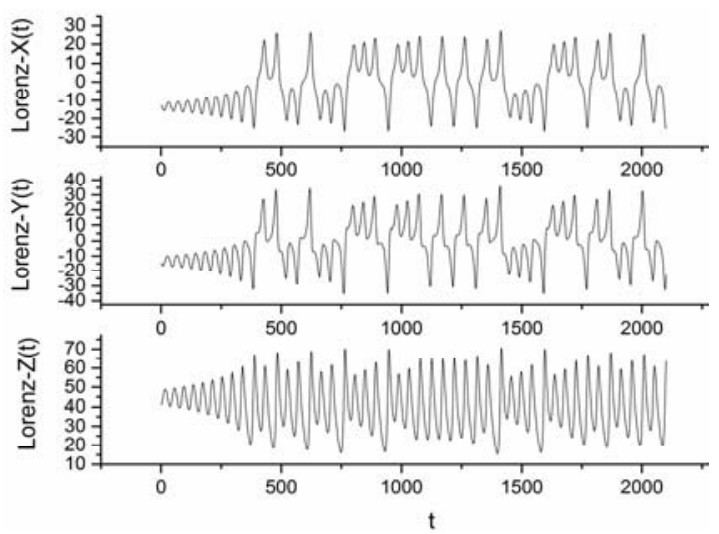

FIGURE II. LORENZ CHAOTIC TIME SERIES ORIGIN DATA GRAPH

\section{B. Multivariate Chaotic Time Series Prediction Experiment}

In this paper, Matlab neural network ntstool box is used to build and train the NARX neural network. The input data for the Lorenz chaotic time series is $\mathrm{x}(\mathrm{t}), \mathrm{y}(\mathrm{t})$ and $\mathrm{z}(\mathrm{t})$. And the output data is $x(t+1)$. In order to determine the parameters of the NARX neural network model of neurons in the hidden layer and the number of delay order, using the expert experience method and trial method to determine the time delay, found that the delay order is 6 , the number of hidden layer neurons is set to 20 , the training get good effect.

Then the NARX neural network is trained to predict the future 100 step by the rolling extrapolation method, and the results are shown in Figure III. From Figure III we can see, the 100 step forecasting Lorenz chaotic time sequence curves and the actual values are in good agreement, the prediction error curve also shows that the maximum prediction error of the prediction is only 0.001 , indicating high prediction accuracy of the model. 


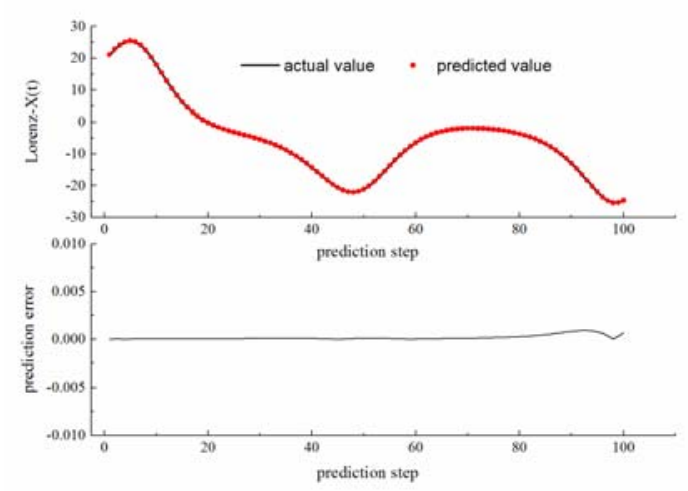

FIGURE III. LORENZ MULTIVARIATE CHAOTIC TIME SERIES PREDICTION AND PREDICTION ERROR

\section{Single Variable Chaotic Time Series Prediction Experiment}

Chaotic time series prediction experiment of single variable and multivariate chaotic time series prediction experiment platform is the same, only single variable chaotic time series prediction experimental input data construction and training of the NARX neural network for chaotic time series is Lorenz' $\mathrm{x}(\mathrm{t})$ single variable data, and the output data is $\mathrm{x}(\mathrm{t}+1)$. Compared with the experimental results of the multivariate chaotic time series prediction, the parameters are the same. The results of single variable of Lorenz chaotic time series prediction are shown in Figure IV.
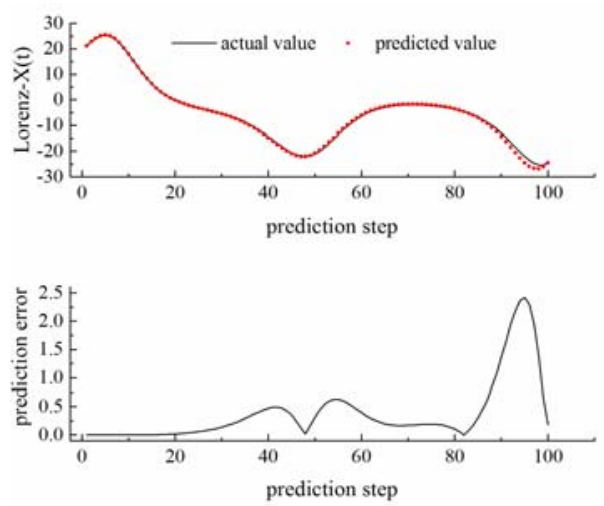

FIGURE IV. LORENZ SINGLE VARIABLE CHAOTIC TIME SERIES PREDICTION AND PREDICTION ERROR

\section{Prediction Result Analysis}

It can be seen from Figure III that the Lorenz multivariate chaotic time series has the absolute prediction error of the maximum value of 0.000938 after the rolling extrapolation of the 100 step. From Figure IV we can see that the Lorenz single variable chaotic time series after rolling 14 step extrapolation, the absolute prediction error value has reached 0.00105 , in the 100 step through rolling extrapolation, the absolute prediction error to a maximum value of 2.4168. From Table I, we can see the forecasting result of the Lorenz multivariate chaotic time series with the natural structure as the input of the model is better than only the forecasting results using the single variable sequence. Comparison of the root mean square error of
MKELM algorithm of reference [11] with the NARX algorithm, we can see that the multivariate NARX neural network prediction algorithm is better than that of MKELM algorithm. A NARX neural network prediction algorithm is more suitable chaotic time series than others.

TABLE I. ROOT MEAN SQUARE ERROR OF LORENZ CHAOTIC TIME SERIES PREDICTION

\begin{tabular}{|l|l|c|}
\hline \multirow{2}{*}{ Model variable } & \multicolumn{2}{|c|}{ Model } \\
\cline { 2 - 3 } & NARX model & MKELM model \\
\hline Multivariate & 0.000295 & 0.0312 \\
\hline Single variable & 0.068 & 0.0973 \\
\hline
\end{tabular}

\section{CONCLUSIONS}

In this paper, a prediction model of chaotic time series with natural multi variable structure is established by using nonlinear autoregressive with exogenous inputs. It has good prediction effect on the Lorenz chaotic time series rolling prediction. Compared with the single variable prediction results of Lorenz chaotic time series, it is proved that the prediction accuracy of multivariate chaotic time series is much higher than that of the single variable time series. Moreover, it is found that the NARX neural network has strong nonlinear mapping ability, and has higher prediction accuracy than other algorithms.

\section{ACKNOWLEDGMENT}

This work has been supported by the General Project of Humanities and Social Science Research of Education Ministry (11YJCZH202).

\section{REFERENCES}

[1] Yang Xueming, Bian Ji Fei, Zhu Xiaoxun, et al. "Study on Support Vector Machine Based on support vector machine for short term wind speed forecasting”. Journal of solar energy, 2016, 37 (2173-2179.): (in Chinese)

[2] Jiang Xiang Cheng. "Hydrological chaotic time series prediction based on Volterra adaptive method”. mathematical statistics and management, 2015, 34 (): 434-441

[3] Du Liuqing, Yin Guofu, Yu Yongwei. "Predicting the motion precision of NC machine tool chaotic phase space reconstruction". Journal of agricultural machinery, based on the 2015, 46 (10): 397-402.

[4] Wang Lan, Li Huaqiang, Wu Xing, et al. "A chaotic time series prediction model for wind power based on improved local Volterra adaptive filter ”. automation of electric power equipment, 2016, 36 (): 40-44.

[5] Wang Shi, Yang Huaijiang, Dong Yan. "To predict the traffic chaos theory based on LAN ". Journal of Beijing Institute of Technology, 2016, 36 (6): 616-619.

[6] Yi Yi, Huang Xueli. "Supply chain performance evaluation based on linear regression forecasting model of chaotic time series". statistics and decision, 2015 (22): 44-46.

[7] Zhang Hongli, Li Ruiguo, fan. "By Bernstein neural network prediction of chaotic sequence of phase space reconstruction ". system simulation, 2016, 28 (4): 880-889.

[8] Han Min, Xu Meiling, Ren Jie. "The state machine for multivariate chaotic time series prediction model ". Acta Automatica Sinica, 2014, 40 (5): 822-829. 
[9] Tanaka Da, Gao x m, Shi Tong. "Combined kernel function least squares support vector machine for chaotic time series prediction”. Acta phys Sinica, 2014, 63 (): 66-76.

[10] Zhang Jinliang, Tan Zhongfu. "Hybrid forecasting method of chaotic time series ”. systems engineering theory and practice, 2013, 33 (): 763769.

[11] Wang Xin Ying, Han min. "Modeling and prediction of multi core extreme learning machine based on multivariate chaotic time series". Acta phys Sinica, 2015, 64 (): 70504-070504.

[12] Math Works. Matlab R2013b Neural Network Toolbox user Guide. 2013 